\title{
Análisis de causas de ineficiencias en servicio al cliente
}

\section{Analysis of inefficiencies in customer service}

\author{
DOI: http://doi.org/10.17981/bilo.2.1.2020.10
}

Artículo de Investigación Científica. Fecha de Recepción: 30/05/2020. Fecha de Aceptación: 14/06/2020

\author{
Valentina Alarcón-Mendoza \\ Universidad de la Costa CUC. Barranquilla (Colombia) \\ valarcon@cuc.edu.co \\ Wendy Sarmiento-Campo \\ Universidad de la Costa CUC. Barranquilla (Colombia) \\ wsarmien9@cuc.edu.co \\ Jaime Mejía-Quiñones \\ Universidad de la Costa CUC. Barranquilla (Colombia) \\ jmejia58@cuc.edu.co \\ Álvaro Castaño-Álvarez \\ Universidad de la Costa CUC. Barranquilla (Colombia) \\ acastano5@cuc.edu.co \\ Alexander Troncoso-Palacio \\ Universidad de la Costa CUC, Barranquilla, (Colombia) \\ atroncos1@cuc.edu.co
}

Para citar este artículo:

V. Alarcón Mendoza, W. Sarmiento Campo, J. Mejía Quiñones, A. Castaño Álvarez, A. Troncoso Palacio “Análisis de causas de ineficiencias en servicio al cliente", BILO, vol. 2, no. 1, 2020. DOI: http://doi.org/10.17981/bilo.2.1.2020.10

\section{Resumen}

En toda compañía es fundamental brindar un buen servicio de calidad a sus clientes, debido a que eso la mantendrá posesionada en los primeros lugares dentro de su sector, también es muy importante resolver todas las peticiones, quejas y reclamos de los usuarios para poder satisfacer sus necesidades, pero cuando se incrementan las quejas y reclamos por un deficiente servicio se hace necesario intervenirlas. Por ello se realizó este estudio, el cual se desarrolló en una empresa donde se tienen unos estándares de atención a usuarios de 8 minutos cada uno. Con base al análisis del diagrama de Ishikawa, se puede notar que las causas de mayor incidencia tienen en la deficiente atención que se les presta a algunos usuarios, son la falta de entrenamiento y las pocas personas en los puestos asignados

Mediante las herramientas de calidad se realizó un proceso de recolección de datos y estudio de posibles causas, con la ayuda del diagrama de causa y efecto se identificó que la causa con más incidencia es la ausencia del personal y falta de capacitación, con las Cartas de Control (por variables o por atributos) se evidenció que se cumplen los estándares de 
calidad establecidos en el tiempo de espera pero el proceso no se encuentra estable y posteriormente con la Capacidad de Proceso se evidenció que la empresa no cumple con las especificaciones establecidas.

Palabras claves: calidad; servicio al cliente; herramientas de calidad; cartas de control; diagrama Ishikawa; capacidad de proceso.

\begin{abstract}
Providing a quality service to its clients in every company is essential to provide a quality service to its clients, it is very important to resolve all the requests, complaints and claims of the users to satisfy their needs and manage to remain possessed in the market. But due to the increase in complaints from users to meet all their needs, but due to the increase in complaints and complaints about the poor service in that area a detailed study of the problem was carried out. The quality tools carried out a process of data collection and study of possible causes, with the help of the cause and effect diagram it was identified that the cause with the most incidence is the absence of staff and lack of training, with the Letters of Control (by variables or by attributes) it was shown that the quality standards established in the waiting time are met but the process is not stable and subsequently with the Process Capacity was shown that the company does not meet the established specifications.
\end{abstract}

Keywords: quality; customer service; quality tools; control charts; Ishikawa diagram; process capacity.

\title{
I. INTRODUCCIÓN
}

La importancia de brindar un buen servicio o atención al cliente radica en los siguientes aspectos: Es necesario ofrecer un valor agregado. Los clientes son cada vez más exigentes, ya no sólo buscan precio y calidad, sino también, una buena atención, un ambiente agradable, comodidad, un trato personalizado, un servicio rápido y posiblemente si un cliente queda insatisfecho por el servicio o la atención, es muy probable que hable mal de la empresa y cuente de su mala experiencia a otros consumidores. El buen servicio debe estar presente en todos los aspectos del negocio en donde haya alguna interacción con el cliente, debe ir desde el saludo del personal encargado de la seguridad del negocio, hasta la llamada contestada por la secretaria. Para ello se debe capacitar a todo el personal, todos deben estar motivados en dar una buena atención, todos deben mostrar siempre un trato amable y cordial con todos y cada uno de los clientes. Y, por último, el buen servicio, no sólo debe darse durante el proceso de compra, sino que también, debe estar presente después de la compra, por ejemplo, al llamar al cliente para conocer su impresión del producto, o al enviarle cartas en su defecto para ofrecer un excelente servicio hasta en la postventa. Para lograr erradicar las inconformidades de los usuarios al momento de la atención al cliente se utilizarán algunas herramientas de calidad como el diagrama de causa efecto, además de las cartas de control (por variables o por atributos) y el cálculo de la capacidad de proceso. Así identificando y evaluando los procedimientos realizados en las operaciones desarrolladas por parte del trabajador y posteriormente se realizarán recomendaciones de mejora, también se determinarán y mejorarán los métodos de trabajo desarrollados en el proceso productivo para ayudar a las empresas a ser más competitivas.

\section{REVISIÓN DE LITERATURA}

En algunos estudios como los realizados por [1,2] se explican que para alcanzar la calidad deben de cumplirse una serie de requisitos. Estos requisitos vienen demandados por el cliente. Debe priorizarse la eficacia en la consecución de dicho objetivo, lo más eficientemente posible y así se alcanzará una gestión efectiva de la organización. Y, según la norma (NC/ ISO 9000 2005) la calidad es entendida como el grado en el que un conjunto de características inherentes cumple con los requisitos. [3, 4] Se puede afirmar que la calidad de un producto viene determinada solo por aquellas características que defina el cliente. La atención al cliente es una modalidad de gestión que empieza desde lo más alto de la organización. Se trata de la filosofía y la cultura de la empresa. Por otra parte, [5] El control puede definirse como la evaluación de la acción, para detectar posibles desvíos respecto de lo planeado, desvíos que serán corregidos mediante la utilización de un sistema determinado cuando excedan los límites admitidos. También puede definirse como la regulación de actividades de acuerdo con los requisitos de los planes. En este artículo se estudiará la problemática que se presenta comúnmente en las empresas y es el deficiente servicio en el área de atención al cliente mediante algunas herramientas de calidad, [6, 7] se puede decir que de la misma manera mencionada anteriormente con el control adecuado de calidad en el área del servicio al cliente se pueden disminuir completamente las informidades de los usuarios y [8] la calidad del servicio no puede ser entendida sin considerar el papel clave jugado por estos últimos. En este sentido, las compañías deben asegurar la gestión adecuada de sus recursos humanos para garantizar la excelencia del servicio, para lo cual, [9] existe una necesidad absoluta de evaluar permanentemente la calidad de los servicios más relevantes prestados por organizaciones públicas y privadas. Se utilizó en primera instancia $[10,11,12]$ el diagrama de causa y efecto también llamado Ishikawa esta herramienta es la representación de las relaciones múltiples de causa - efecto entre las diversas variables que intervienen, se evidencia el efecto en la cabeza del diagrama y en su cuerpo las diferentes causas y subcausas que participan en el proceso; luego de esto se realizaron las cartas de control para estudiar la variabilidad a través del tiempo, lo cual es clave para mejorar los procesos, mediante tres actividades básicas como lo estabilizar los procesos 
(lograr control estadístico) en la medida en que se detectan, identifican y eliminan las causas especiales de variación, mejorar el proceso al reducir la variación debida a causas comunes y monitorear el proceso para asegurar que las mejoras se conserven y para detectar oportunidades adicionales de mejora, [13, 14, 15] Por último se analizó la capacidad del proceso que consiste en conocer la amplitud de la variación natural del proceso para una característica de calidad dada, esto permitirá saber en qué medida tal característica de calidad es satisfactoria.

\section{METODOLOGÍA}

Se estudiarán las deficiencias de calidad en el área de atención al cliente. Para la carta de control X-R se tomarán 10 muestras de tamaño 10 midiendo el tiempo de atención de cada usuario para constatar que se cumplan estos tiempos requeridos en atención, de no ser así se podrá sugerir la asignación de más funcionarios a ventanilla o capacitar al personal para que sean más rápidos, en caso contrario de terminar la atención antes de 8 minutos se podrá disminuir el número de personal en ventanilla. También se analizaron las causas de las inconformidades de los usuarios, para lograr dar una solución a la problemática. Para lo anterior se utilizará el diagrama de Ishikawa.

\section{DESARROLLO}

Para la carta de control X-R se tomarán 10 muestras de tamaño 10 midiendo el tiempo de atención de cada usuario para constatar que se cumplan estos tiempos requeridos en atención, de no ser así se podrá sugerir la asignación de más funcionarios a ventanilla o capacitar al personal para que sean más rápidos, en caso contrario de terminar la atención antes de 8 minutos se podrá disminuir el número de personal en ventanilla. Mediante el diagrama de Ishikawa se obtuvo la información de causas y sub causas principales de la deficiencia en la atención al usuario, con el fin de analizar el servicio y mejorar la calidad de este para que se reduzcan las peticiones quejas y reclamos, aumentando la eficacia del servicio y mejorando la imágen de la empresa [16-17]

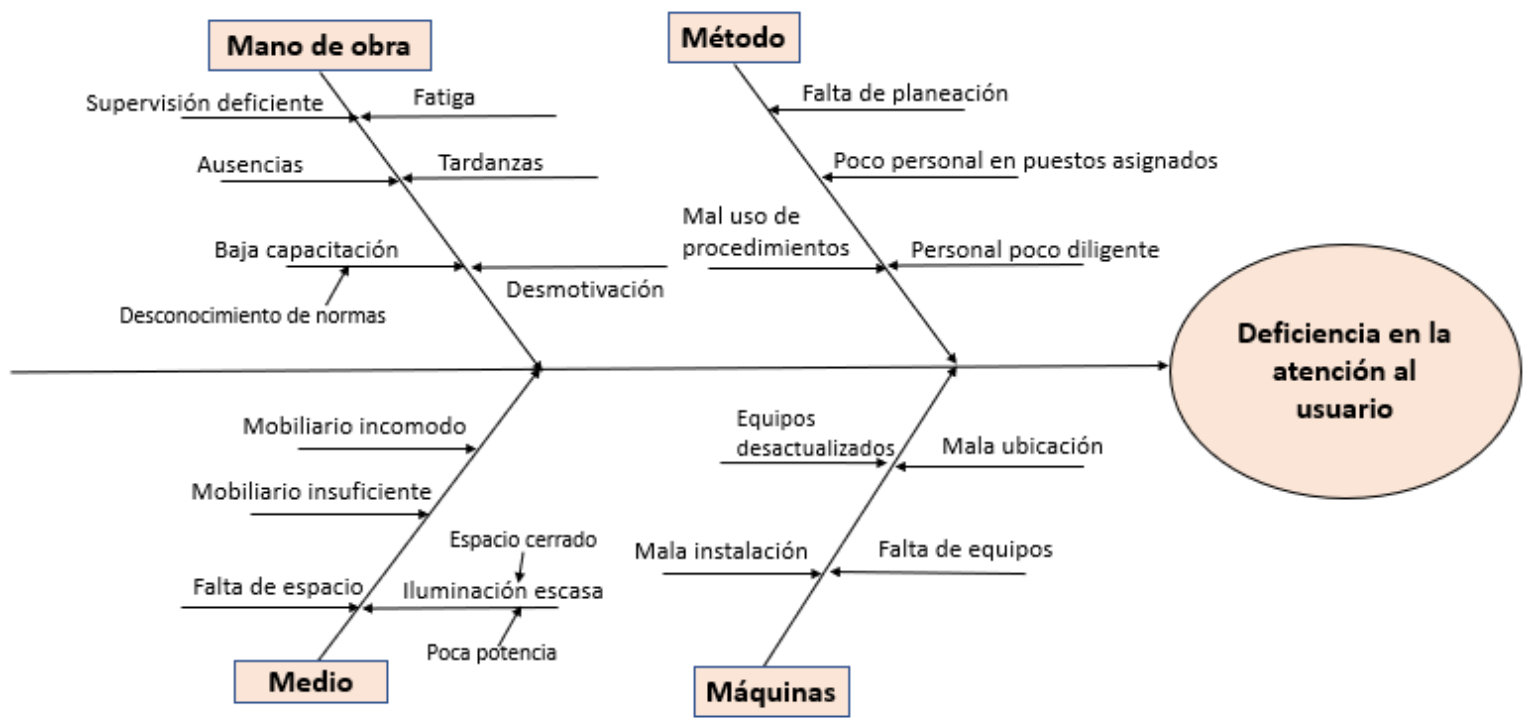

Figura 1. Diagrama causa y efecto

Con el diagrama de Ishikawa diseñado se clasificaron las causas principales y sub causas, que generan que el servicio prestado a los clientes sea deficiente. Se identifica que las causas vitales son caja capacitación del personal, mal uso de procedimientos, poco personal en puestos asignados. Por lo que se debe hacer énfasis en ellas con un plan de acción.

\begin{tabular}{ccccccc}
\hline \multicolumn{3}{c}{ Datos de medias } & \multicolumn{3}{c}{ Datos de rangos } \\
$\mathbf{X}$ & LCS & LCI & LC R & LCS & LCI \\
5,586000 & 7,6804 & 3,4916 & 6,8 & 12,0836 & 1,5164 \\
\hline
\end{tabular}

Tabla 1. Valores de los límites de control. 
Para medir los tiempos se usó una carta por variable X - R, con este procedimiento se quiere determinar si la empresa cumple con los estándares que tiene establecidos, y si se puede darle una mejora. Teniendo una vez lo datos de los límites de control se procede a graficar:

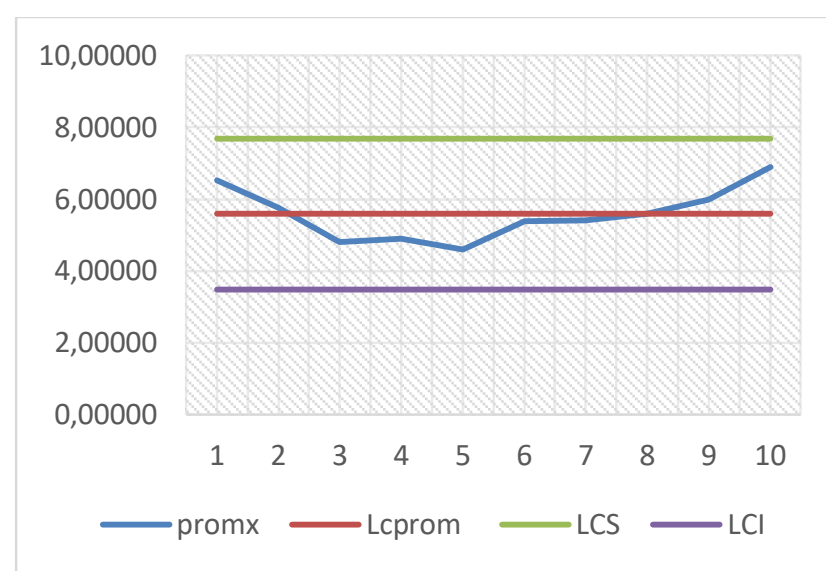

Figura 1. Gráfico de control de medias

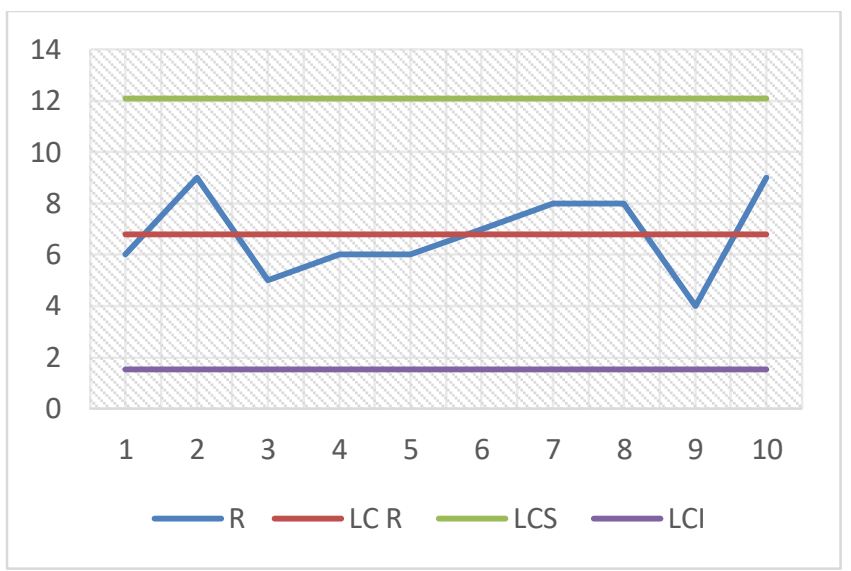

Figura 2. Gráfico de control de rangos

Al analizar las gráficas se evidenció que la empresa cumple con los estándares de calidad con respecto al tiempo que determinan ellos necesarios para que un usuario pueda ser atendido. Y podemos decir que es una empresa que está en una posible constante mejora para que sus clientes se encuentren satisfechos con el servicio prestado. Luego de ello se realizó el cálculo respecto a la capacidad de este proceso:

$$
\begin{gathered}
\sigma=\frac{6,8}{3,078}=2,209 \\
C p=\frac{7,7204-3,5316}{6 \times 2,209}=0,31604
\end{gathered}
$$

En la capacidad podemos observar que el proceso que sigue la empresa no cumple con las especificaciones

\section{v. CONCLUSIONES.}

Teniendo en cuenta que a pesar de que la empresa cumple con los estándares de calidad establecidos en el tiempo que puede permanecer un usuario en espera, el proceso que establece no se encuentra estable. Con base al análisis del diagrama de Ishikawa, se puede notar que las causas de mayor incidencia tienen en la deficiente atención que se les presta a algunos usuarios, son la falta de entrenamiento y las pocas personas en los puestos asignados. Por tanto, la Empresa debe implementar un plan de capacitación del personal para que pueda entablar una comunicación más efectiva con el usuario, con el fin de satisfacer su necesidad de manera más rápida. Teniendo en cuenta que algunas veces se presentan usuarios que no saben redactar o explicar lo que necesitan, u otros que llegan realizando peticiones con mejor importancia como por ejemplo que le quiten una publicidad que viene en su recibo que no le está generando ningún costo, pero simplemente él no quiere que le aparezca y eso genera tiempo innecesario, y demoras para los demás usuarios. Por lo tanto, es necesario que el área de talento humano se comprometa con la capacitación del personal, para lograr reducir las peticiones, quejas y a su vez reducir costos en los procesos de mejora. También se debe implementar una excelente logística, agilizando procesos no esperar que la sala de espera llegue al límite máximo de personas para que puedan asignar más usuarios, es decir, estar asignado personal rotativamente para cumplir con las especificaciones en el proceso.

Con ayuda de las herramientas de calidad aplicadas en este estudio se evidencia que la empresa presenta fallas en cuanto al personal capacitado para cumplir con las tareas y compromisos establecidos por cada área. Por ello es recomendable realizar cursos de capacitaciones a todo el personal para mejorar los procedimientos y además disponer del correcto personal para cada puesto, debido que no hay suficiente personal para realizar las labores, por tal motivo hay ausencia en los puestos de atención al usuario y esto hace que el trabajo sea menos eficiente.

\section{REFERENCIAS.}


[1] Escuela Europea de Excelencia, «Calidad total: definición y conceptos fundamentales,» 2019.

[2] International Organization for Standardization, «https://www.iso.org/obp/ui/\#home,» 2015. [En línea]. Available: https://www.iso.org/obp/ui/\#iso:std:iso:9000:ed-4:v1:es. [Último acceso: 225 2020].

[3] D. Tigani, Excelencia en servicio, B. V. CUNORI, Ed., Liderazgo 21, 2018.

[4] A. S. Toledo Andrade, M. N. Varela Vielma y P. M. Cabeza García, «Revisión de pautas en la implementación de estrategias inclusivas de servicio basadas en el cliente interno y externo,» Revista metropolitana de ciencias aplicadas, vol. 3, n 2, pp. 90-97, 2020.

[5] F. O. Mayorga Mayorga y K. M. Manzano Chifla, «Sistema de gestión de relación con el cliente (CRM) para mejorar la calidad de servicio al cliente en instituciones financieras reguladas por la SEPS,» Repositorio Digital Universidad Técnica de Ambato, 2020.

[6] M. A. Sullo Rosello, «5s para mejorar la gestión de almacenes y el servicio al cliente en empresas industriales, Lima 2019,» Repositorio Digital de la Universidad César Vallejo, 2020.

[7] M. Torres Samuel y C. L. Vásquez stanescu, «Modelos de evaluación de la calidad del servicio: caracterización y análisis,» Compendium, vol. 18, nº 35, pp. 57-76, 2015.

[8] A. Orgambídez Ramos y M. H. De almeida, «Antecedentes organizacionales de la calidad de servicio: un modelo de relaciones estructurales,» Redalyc.org, pp. 167-173, 2015.

[9] F. J. Torres y E. I. Luna, «Evaluación de la percepción de la calidad de los servicios bancarios mediante el modelo SERVPERF,» ScienceDirect, 2017.

[10] E. F. Novillo Maldonado y J. N. Preciado Serrano, «Aplicación de diagrama causa-efecto en una institución de educación superior: caso Universidad Técnica de Machala.,» Repositorio Digital de la UTMACH, 2019.

[11] L. Pérez Noda, O. De la Cruz Rivadeneira y F. Marrero Delgado, «Organización de empresas por procesos. Caso de estudio: Empresa Pesquera Sancti Spíritus,» Revista Científica Infociencia, vol. 19, nº 4, 2015.

[12] C. Ortiz Hernandez, A. Troncoso Palacio, D. Acosta Toscano, R. Begambre Meza y B. Troncoso Mendoza, «Utilización de Herramientas de Calidad para la Mejora en los Procesos de Extrusión de Plásticos,» Boletín de Innovación, Logística Y Operaciones, vol. 1, nº 1, pp. 1-7, 2019.

[13] G. Herrera Vidal, Y. Pérez Aguas y E. Venecia Puello, «Enfoque seis sigma y proceso analítico jerárquico en empresa del sector lácteo,» Revista Venezolana de Gerencia, vol. 22, nº 80, pp. 610-636, 2017.

[14] A. Bohigues Ortiz y V. Gisbert Soler, «Desarrollo e implementación de un modelo Seis Sigma para la mejora de la Calidad y de la productividad en Pymes industriales,» Universidad Politecnica de Valencia, 2015.

[15] M. J. Ortíz Tovar, «Implementación del modelo Six Sigma como estrategia de mejora en Pymes de Latinoamérica,» Fundación Universidad de América, 2020.

[16] Bertolli, M., Roark, G., Urrutia, S., \& Chiodi, F. (2017). Revisión de modelos de madurez en la medición del desempeño. INGE CUC, 13(1), 70-83. https://doi.org/10.17981/ingecuc.13.1.2017.07

Rodríguez, L., Castellano, M., \& Caridad, M. (2017). Planificación estratégica de recursos humanos en

[17] empresas de consumo masivo. IJMSOR: International Journal of Management Science \& Operation Research, 2(1), 38-43. Recuperado a partir de http://ijmsoridi.com/index.php/ijmsor/article/view/84 